\title{
The hormonal mechanism of heat stress effect on the carbohydrate metabolism in Drosophila melanogaster females
}

\author{
M.A. Eremina*, E.K. Karpova, N.E. Gruntenko \\ Institute of Cytology and Genetics SB RAS, Novosibirsk, Russia \\ *e-mail:eremina@bionet.nsc.ru
}

Key words: heat stress, dopamine, octopamine, juvenile hormone, 20-hydroxyecdysone, Drosophila

The Drosophila studies have revealed a strong evolutionary conservatism of the insulin/ insulin-like growth factor-like signaling (IIS) and its involvement in the regulation of metabolic homeostasis and resistance to various types of stressors. The two main forms of circulating carbohydrates in Drosophila are glucose and trehalose (glucose disaccharide). The use of the evolutionary conservatism of I/ IGF makes it possible to analyze the mechanisms underlying the development of diabetes mellitus and allows the experimental study of the influence of factors that can have a provoking effect on it, in cases where human research is impossible. One of these factors is stress - the universal response of living organisms to any adverse influences. Various hormones have been implicated in the stress response of the Drosophila adult, in particular biogenic amines dopamine (DA) and octopamine (OA), which perform neurotransmitter, neuromodulatory and neurohormonal functions, juvenile hormone $(\mathrm{JH})$ and 20 -hydroxyecdysone $(20 \mathrm{E})$, playing a fundamental role in the control of reproductive function in adult insects. The purpose of this work is to study the effect of heat stress on the carbohydrate metabolism of D. melanogaster females of the wild type Canton S (CS) strain combinated with changes in the level of stress-related hormones (DA, OA, JH, 20E). We show that:

1) $\mathrm{DA}$ and $\mathrm{JH}$ have an inhibitory effect on carbohydrate metabolism under normal conditions;

2) OA and 20E exert a stimulating effect on the level of the main carbohydrate of insects, trehalose, but an inhibitory effect on the level of glucose under normal conditions;

3) DA, JH, 20E have an inhibitory effect on the level of both carbohydrates, OA has an inhibitory effect on the level of glucose and a stimulating effect on the level of trehalose in case of heat stress.

Our data suggest that the hormones involved in the neuroendocrine stress response of D. melanogaster are involved in the regulation of carbohydrate metabolism. Further work will focus on correcting carbohydrate metabolism in D. melanogaster with strains disrupted IIS disorders. 